\title{
How to see a redder red
}

\section{Engineered pigment absorbs wavelengths undetectable by the natural human eye.}

\section{Amy Maxmen}

\section{December 2012}

When Bob Marley sang, "I am redder than red," he probably did not imagine that chemists would one day capture this imagined hue. But researchers have taken a step in that direction, by tweaking a colour-sensing pigment from the human eye to absorb reds of longer wavelengths than those that we can see.

"We didn't expect to get redder than red," says Babak Borhan, a chemist at Michigan State University in East Lansing, who led the study published today in Science ${ }^{1}$.

The researchers' red was the product of experiments conducted to understand exactly how colour-sensing pigments in our eyes absorb different hues. The team targeted rhodopsin, a pigment found in the photoreceptor cells of the retina.

A rhodopsin molecule is made of proteins called opsins and a chromophore - the part of the molecule responsible for absorbing different wavelengths of light . Together, the two parts translate light into signals for various colours, which are then interpreted by the brain. In the eye, a chromophore called retinal responds to wavelengths ranging from red, at about 560 nanometres, to blue, at about $420 \mathrm{~nm}$.

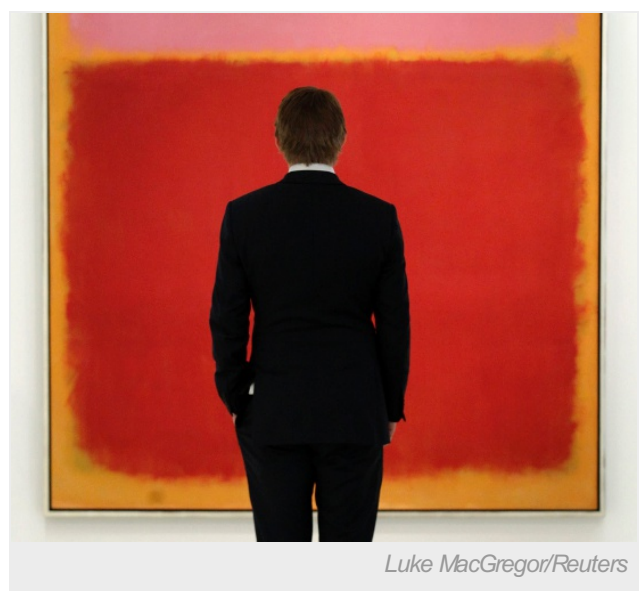

As red as it gets: this Christie's employee gazing at Mark Rothko's "Untitled \#17" in London, 2011, would be unable to see the redder reds which engineered pigments can now absorb.

"The question has been: how can we see all of these colours using essentially one molecule, the chromophore?" Borhan says.

The attached proteins somehow control the range of light that a chromophore can absorb — from red, to green, to blue; but no one knew exactly how they fine-tuned this absorption ability. Scientists have hypothesized that the shade the chromophore can receive shifts as a result of more than one interaction, such as a change in the shape of the chromophore-protein complex and a change in the positions of electrical charges along the protein molecule.

"In order to get a red shift, people thought you might need to apply a combination of factors, like a massive tug of war with all of the players pulling in the same direction," says Thomas Sakmar, a chemical biologist at Rockefeller University in New York. However, Borhan's work now shows that only one factor is needed to change a chromophore's sensitivity to light.

\section{Life in the red}

Borhan's team tinkered with the amino acids in a construct made of retinal and an engineered protein surrogate for opsin, to help reveal which interactions pushed the chromophore furthest in its ability to absorb different wavelengths of light.

Some amino acids carry electrical charges, and normally a positive charge localizes to one spot on the molecule. But the team was able to distribute, or "blur" this positive electrical charge evenly across the construct by mutating amino acids in the protein.

And this engineered pigment absorbed far 'redder' wavelengths - $644 \mathrm{~nm}$ - than a natural red-sensing rhodopsin would 'see'.

"What's interesting is they didn't cause the chromophore to twist [change shape] at all — they showed you could get a red shift by neutralizing a positive charge," Sakmar says. "I think people will now want to engineer new pigments on the basis of this work. To me, that's the definition of a cool paper — people will use it."

For Borhan, one tantalizing detail remains elusive: a glimpse into what life looks like with photoreceptors that absorb 644-nm wavelengths of light. "I guess we'd see far into red, but not so red that we'd see heat signatures," he says. "We'd see reds in greater definition, but really, I can't know for sure." 


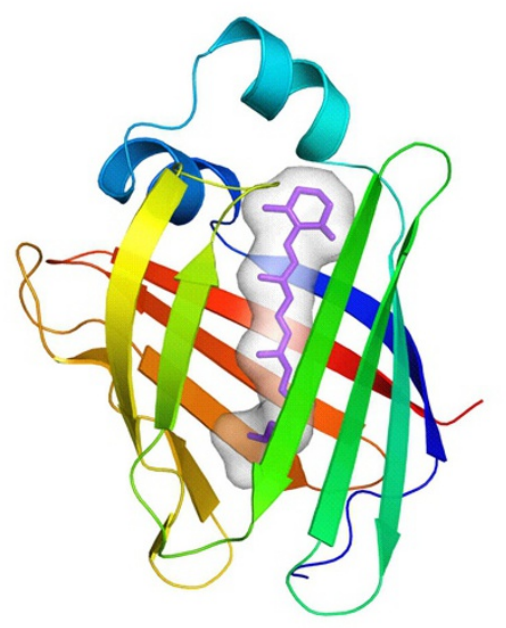

Engineering the structure of the photoreceptor to spread out positive electrical charges seemed to enable "super-red" vision. The chromophore is central, and the protein shown around it.

Nature | doi:10.1038/nature.2012.11973

\section{References}

1. Wang, W. et al. Science 338, 1340-1343 (2012). 